Gut, 1962, 3, 267

\title{
Interstitial emphysema of the caecum
}

\author{
J. J. KAY-BUTLER
}

From the X-ray Department, Central Middlesex Hospital, London

EDITORIAL SYNOPSIS A case of intestinal emphysema localized to the caecum is reported together with an experiment to try and reproduce the condition in vitro.

Interstitial emphysema in the wall of various parts of the alimentary tract is a rare finding. Koss (1952) and Faggella, Dewar, and Schilling (1960) have collected series of cases but the mechanism has not been established with certainty.

\section{CASE REPORT}

A 56-year-old man, A.B., was admitted to the Central Middlesex Hospital on 18 June 1957 complaining of diarrhoea for six weeks and loss of weight and strength for about two months.

His past history included a total gastrectomy six months previously for a leather bottle type carcinoma of the stomach and subsequently he had developed a leftsided empyema followed by a lung abscess at the apex of the right lower lobe; on both occasions he was readmitted to hospital. He had only been at home one month before the present admission.

On examination he was found to be very cachectic and his haemoglobin was $64 \%$. The severe diarrhoea was causing malabsorption and steatorrhoea ( $33 \mathrm{~g}$. per day). He had a resonant mass in the right iliac fossa which came and went during examination. There were nodules in the subcutaneous tissues close to the gastrectomy scar. A barium meal on 27 June showed a normal oesophagojejunostomy but the follow-through examination suggested incomplete obstruction of the transverse colon. A carmine marker gave a transit time of eight hours from mouth to anus.

His treatment was conservative in view of the probability of widespread secondaries but he showed considerable improvement on sulphonamides and the diarrhoea stopped. Suddenly on 1 July he complained that 'something has burst inside' and a radiograph of the abdomen showed gas in the wall of the caecum (Fig. 1) and under the diaphragm. The gas in the caecal wall was in a narrow radiolucent band completely outlining the caecum, which contained much faecal matter. A perforation was diagnosed and in view of the malignant nature of his disease conservative treatment was adopted. He collapsed and died a few hours later.

At post-mortem examination 40 hours after death extensive carcinomatous involvement of the caecum, peritoneum, intestinal wall, and abdominal glands was shown. A perforation was found in the caecum which measured $1 \mathrm{~cm}$. in the mucous coat and $3 \mathrm{~cm}$. in the peritoneal coat. Gas was not evident to the naked eye in the caecal wall and further efforts to demonstrate it were not made.

\section{EXPERIMENTAL EVIDENCE}

It was decided to try to produce experimentally the appearances noted in this patient, and four fresh postmortem specimens consisting of the caecum and a short length of terminal ileum were obtained. Three short incisions were made in the mucosa of the caecum and $4 \mathrm{ml}$. of barium suspension was introduced into the lumen and the caecum was tensely distended with air. When a certain degree of distension was obtained air spread suddenly through the tissue planes of the caecal wall (Fig. 2). If the caecum was rotated so that the barium was in contact with the incisions barium also permeated the tissue planes. With gentle massage of the caecal wall, simulating peristalsis, air and barium were spread easily in the tissues.

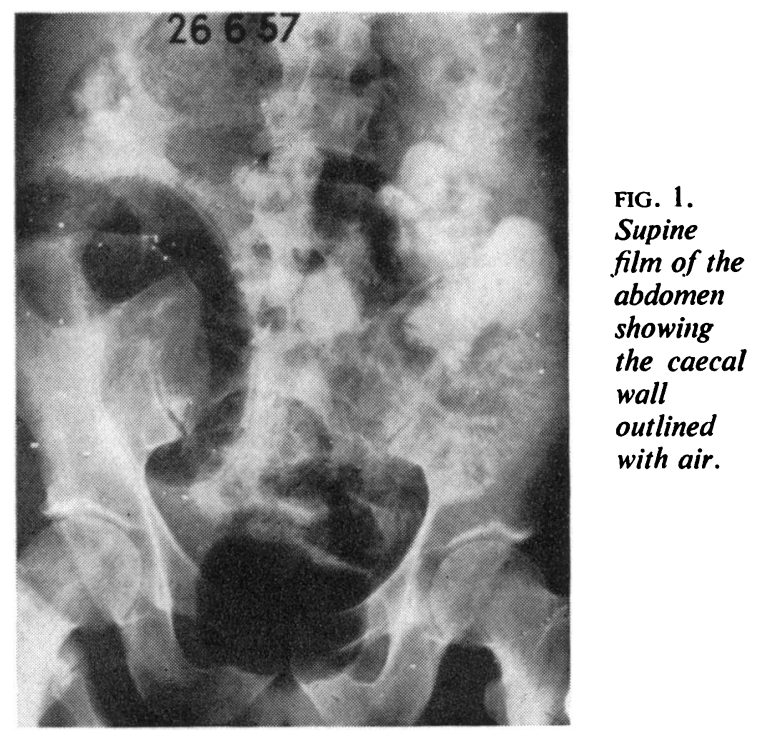




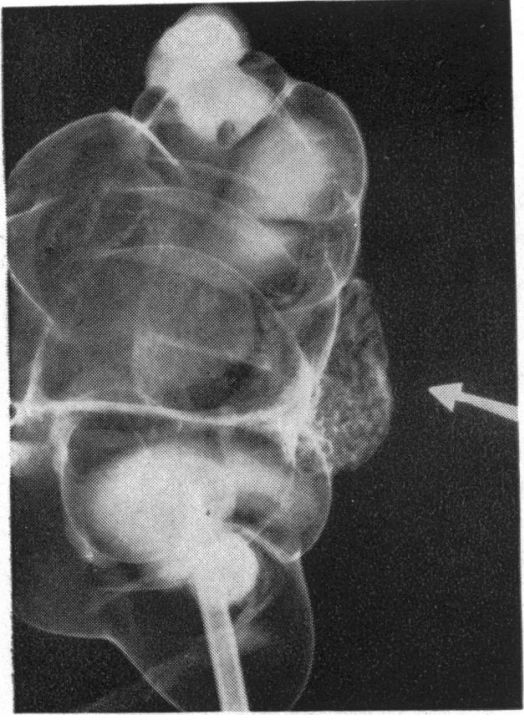

FIG. 2.

Specimen of caecum, tied at the ascending colon and terminal ileum, inflated with air, showing a large bleb that developed (arrowed).

A similar experiment was performed by Hindmarsh (1953) on one caecum which he left for 24 hours in a saline bath inflated to a pressure of $65 \mathrm{~mm}$. $\mathrm{Hg}$. A few bubbles appeared near the incisions but he did not consider it conclusive. A constant pressure was not used in the experiments described here as it was found that the pressure dropped slowly due to various leaks. Instead, the caecum was inflated until interstitial emphysema occurred.

\section{COMMENT}

The radiographic appearance of the caecal wall is thought to be due to submucosal gas spreading through a tear in the mucosa which occurred before gas finally escaped through all coats into the peritoneum.

Stiennon (1951) describes two cases in newborn infants, who suffered from diarrhoea and bleeding from the anus, in whom a 'spectacular' radiolucent stripe outlined the greater part of the colon on all its margins. The translucency, as in the case reported, lay between the mucosa and the mucularis. Stiennon thought that the absence of the typicalcystic accumu- lations of gas characteristic of pneumatosis intestinalis was due to the ease with which the mucosa could be stripped in an infant. Stiennon states that gas, if under pressure, can leave the stomach, duodenum, terminal ileum, or colon and track back to the mesenteric root. Once there the gas can track along the mesentery to the mesenteric insertion of any part of the intestine and from there it spreads subserosally or submucosally by following the blood vessels.

Marshak and Eliasoph (1956) described cases in the left colon which had followed upon sigmoidoscopy and concluded that direct trauma initiated the condition and then the appearances stayed constant for years. Their cases were different in appearance from ours as they were of large, gas-filled cysts in grape-like clusters of various sizes which did not rapidly reabsorb. It seems certain that they were really a closely similar condition, pneumatosis coli. In the present case there was no obstruction at the pylorus as in many of the reported cases in which the gas is considered to reach the colon wall after leaving the stomach by way of the mesentery. Here it travels by way of lymphatics or tissue planes. It is much more likely that the mechanism of production of the two types of appearance is quite different and that when the gas is in a continuous line it has developed due to a break in the mucosa at some point in the intestinal tract, allowing the formation of surgical emphysema.

I am indebted to Dr. F. Pygott for his advice, and to Dr. F. Avery Jones, the physician in charge of this case. The prints from the radiographs were made by $\mathrm{Mr}$. A. Booker.

\section{REFERENCES}

Faggella, R. M., Dewar, J. P., and Schilling, J. A. (1960). Pneumatosis cystoides intestinalis: Report of two cases and review of the natural history of the disease. Amer. J. Surg., 99, 283-291.

Hindmarsh, F. D. (1953). Gas cysts of the intestine. Brit. J. Surg., 41, 70-72.

Koss, L. G (1952) Abdominal gas cysts (Pneumatosis cystoides intestinorum hominis). A.M.A. Arch. Path., 53, 523-549.

Marshak, R. H., and Eliasoph, J. (1956). Pneumatosis coli. Amer. J. dig.Dis., n.s. I, 99-107.

Stiennon, O. A. (1951). Pneumatosis intestinalis in the newborn. Amer. J. Dis. Child., 81, 651-663. 